Journal of Advanced Research in Materials Science

\title{
The Effect of Nanoparticles in Augmentation of Cooling Tower Heat Dissipation
}

\author{
Aravinthan Rajaandra ${ }^{1}$, Nor Azwadi Che Sidik ${ }^{1,}{ }^{*}$, Siti Nurul Akmal Yusof ${ }^{1}$, Muhammadu Masin \\ Muhammadu² \\ 1 Malaysia - Japan International Institute of Technology (MJIT), University Teknologi Malaysia, Jalan Sultan Yahya Petra,54100 Kuala Lumpur, \\ Malaysia \\ 2 Department of Mechanical Engineering, Federal University of Technology, P. M. B. 65, Gidan-Kwanu Minna, Nigeria
}

\begin{abstract}
Cooling towers with water as the heat transfer medium are commonly used in various industries for rejecting heat from heat sources to the atmosphere. However, there is much room for improving the performance and efficiency of the cooling towers. One of the less investigated ways in using a water based nanofluid as the heat transfer medium. Nanofluids which are base fluids with nanoparticles added to them has many benefits including increasing the heat rejection and increasing the range of the cooling tower. This has the effect of lowering energy usage and reducing water consumption. In this study, the cooling tower heat rejection process in the infill was simulated using water as the base working fluid, and Multi Walled Carbon Nano Tube (MWCNT)-water nanofluids. It was observed that the heat rejection was improved by up to $40 \%$ using 0.1 wt $\%$ of Multi Walled Carbon Nano Tube (MWCNT)-water nanofluid. It was found that there was an improvement in heat rejection. It can reduce energy usage by up to $20 \%$ and reduces energy costs using a similar amount. The results indicate that nanofluids may allow existing and future cooling towers to achieve an improvement in heat rejection performance.
\end{abstract}

Keywords:

cooling tower; nanofluid; thermal efficiency

Copyright $@ 2020$ PENERBIT AKADEMIA BARU - All rights reserved

\section{Introduction}

In recent years, sustainable energy and resource usage has become a priority for all nations and industrial sectors. In cooling systems, where energy usage is significant, designing energy efficient systems has become critical in terms of financial viability and marketability. It is well known that fluid flow and heat transfer in such devices can play an important role in energy and environmental technologies [1]. Systems which use sensitive resources such as water to function have become focal points. The increase in environmental and sustainability awareness, energy and water usage efficiency have become important. Heat dissipation is important in many fields. Removing heat energy from a specific load and transferring it to the atmosphere is the fundamental of cooling technology. The efficiency at which this process can occur is paramount as it an energy intensive process. In the case of cooling towers, there is significant room to improve the energy usage and

\footnotetext{
* Corresponding author.

E-mail address: azwadi@utm.my (Nor Azwadi Che Sidik)
}

https://doi.org/10.37934/arms.67.1.1125 
reduce the water consumption [2]. A cooling tower is essentially a device which cools down a stream of fluid by rejecting the heat from the fluid to the atmosphere by transferring the heat energy to atmospheric air [3].

Cooling towers work by the interaction of cooling atmospheric air with the hot water. The air cools the working fluid through convection. Some heat rejection also occurs through evaporation of the working fluid. The diagram in Figure 1 below depicts the working of a typical cross flow cooling tower.

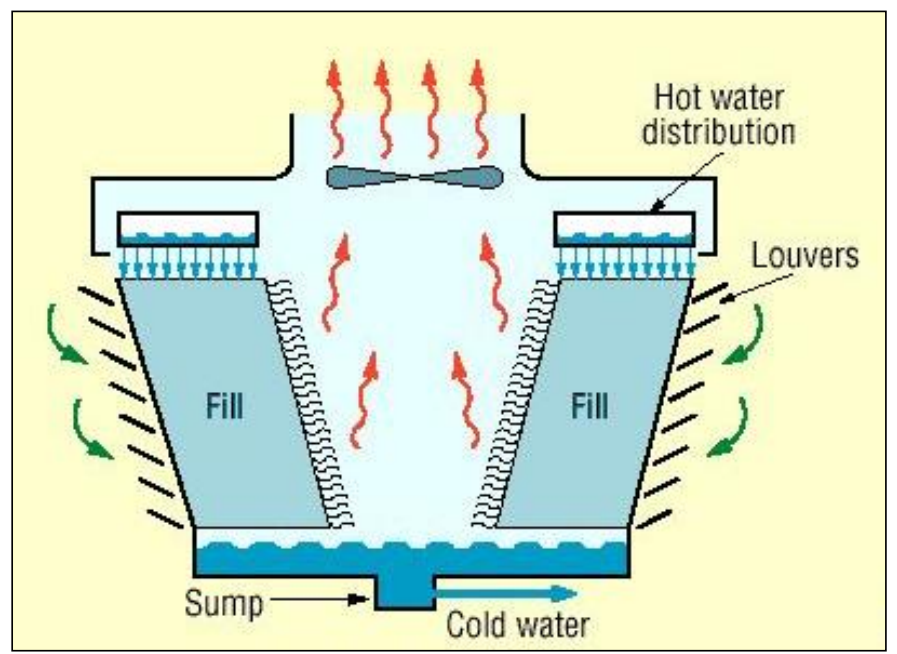

Fig. 1. Typical cooling tower diagram

Cooling towers can be found widely used in various industries where removing heat from processes or building is required, such as chemical plants, refineries, nuclear power plants, office buildings, airports, or shopping malls. The interaction between the hot water and the cooling air is referred to when categorizing the cooling tower as a wet or dry type [4]. Cooling towers are used in centralized cooling systems to dissipate heat energy from the building/plant to the atmosphere using water as the transfer medium. In recent years, Malaysia has experienced higher average atmospheric wet and dry bulb temperatures up to 2 degrees Celsius. As a result, building heat loads are higher, and the effective dissipation envelope for cooling towers is narrower. Studies show that the largest contributor of energy use in large buildings is the cooling system [5], up to 57\%. The breakdown of energy use determined by the paper is shown in Figure 2 .

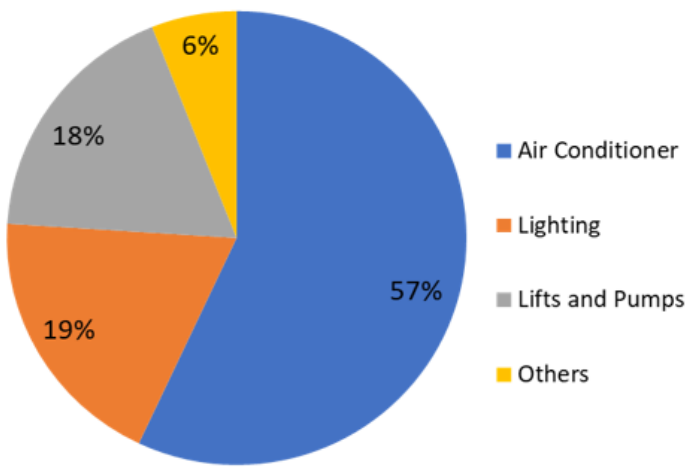

Fig. 2. Energy use in buildings 
While the percentage of energy use for cooling system is significant, the trend in overall energy use in Semenanjung Malaysia [6] has also been upward for many decades. The combination of the overall energy use which is rising, and the percentage of energy use for cooling which is highly significant, and the continuous rise of energy costs lead to one conclusion. Energy costs for cooling buildings will continue to rise, and as cooling towers contribute a large portion of the energy use, any improvement in heat rejection efficiency of cooling towers is likely to have significant monetary impact for the building owners.

Cooling towers are a large market segment and are in widespread use. The cooling tower market worldwide is expected to reach 3.25 billion US Dollars by 2020 [7]. With the expected rise in global temperatures, the demand for cooling is expected to also increase. This will in turn lead to an increase in energy demand and increase in cost spent on energy globally [8].

Furthermore, the environmental, social, and regulatory constraints faced by all technologies including cooling technologies are becoming more stringent daily. As for cooling towers, while several improvements have been made to the various components of the equipment, the fundamental aspect remain stagnant.

The biggest problem in cooling towers is the energy consumption. Being a mechanical contraption, the operation of cooling towers, requires significant energy use. The energy use however is dependent on the mechanical efficiency of the equipment and the efficiency of heat rejection process. The mechanical efficiency has been improved throughout the years by improving the materials, tolerances, and construction quality. The same has been done for heat rejection efficiency by improving the fill material and design, and the distribution of the hot water. However, the fundamental aspect of the process, the heat transfer medium which is water remains the same. There are many possible avenues to improve cooling tower performance and efficiency. Increasingly extensive research has been carried out on the various possibilities in the last two decades or so. Several teams have carried out experimental work focused on packing material or filled beds.

Gharagheizi et al., [5] studied the performance improvement of cooling tower with two different types of film packing. In a similar investigation, Li et al., [9] were able to build a mathematical model for the evaporation and cooling efficiency of counter flow film type cooling tower with film type packing. Their on-site test included heat and mass transport mechanism of film type packing. Goshayshi and Missenden [10] compared the impact of different arrangements of fills in cooling towers. The heat rejection behavior of cross flow cooling towers with splash type filled beds through variable wet bulb temperatures was studied by Hajidavalloo et al., [11].

Beyond packing, some teams concentrated on spray nozzles and the impact of different types of spray nozzles [12]. Overall, most of the studies on cooling tower and other heat exchanger performance enhancement have focused on the impact of the operating conditions and parameters. These include flow rate, water temperature, air temperature and relative humidity, filler type, and dimensional characteristics of the cooling tower. While the results valid and encouraging, some of the parameter modifications will not be implementable industry wide or be cost effective to achieve. The entire process efficiency of heat rejection in the cooling tower is limited by the thermal properties of water.

In the last decade, there has been ample focus on adding nanoparticles to various base fluids for various intents [13]. The addition of Ti/O2 nanoparticles to enhance the heat transfer properties of the medium in solar collectors has shown potential [14]. Peyghambarzadeh et al., [15] introduced Al203-water nanofluids to a vehicle radiator to study the heat transfer coefficient. Compared to just using water, the heat transfer coefficient improved by $45 \%$ post nanoparticle introduction. In a study very close to our objective, Imani et al., [16] experimentally investigated the thermal performance 
improvement of a wet cooling tower using $\mathrm{ZnO}$ /water nanofluid with various arrangements of filled beds.

In solar collector application, adding nanoparticles to the working fluid water has improved the thermal conductivity of the water making it a better heat transfer fluid. Introducing nanoparticles into conventional coolants (water/ethylene glycol, etc.), has been considered for real world applications for at least two decades [14].

One example would be the introduction of $\mathrm{Al} 2 \mathrm{O} 3$ in car radiator cooling water by Peyghambarzadeh et al., [15] to determine the heat transfer coefficient on the tune side. In turbulent conditions, a $45 \%$ improvement heat transfer was recorded. In a study by Lee [17], it was reported that the efficiency of domestic water heat exchanger system was optimum when $1.5 \%$ nanoparticles were added to the water. Numerical investigation of the heat transfer in the domestic water heat exchanger with Copper ( $\mathrm{Cu}$ ) nanoparticles and alumina (Al2O3) nanoparticles were considered at volume fraction ranges $0.5-3.0 \%$.

Askari et al., [18] concluded that the improvements are achieved thermal conductivity of the nanofluids is enhanced with higher temperatures. Inter-particle and inter-molecular adhesion forces are weaker which leads to an increase in Brownian motion. With the increase in random motion of particles, the overall fluid has better thermal conductivity properties. This gives better heat dissipation.

Furthermore, the presence of nanoparticles also increased the contact angle. As established by Lim et al., [19], contact angle is inversely proportional to wettability. With lowered wettability, the surface tension of the fluid rises as found by Bhuiyan et al., [20]. This would account for the reduction of water consumption in the cooling tower. The evaporation loss of water is reduced and the need to replenish the fluid reduced.

Based on the studies reviewed, two things are clear. The use of nanofluids in heat transfer applications has multiple potential benefits. On the other hand, the application of nanofluids in cooling towers, especially locally has to be studied further. There have been several studies conducted on the possibility of replacing water with other nanofluids. Askari et al., [18] published a set of interesting results whereby an improvement of $40 \%$ was achieve in cooling range by using multi-walled carbon nanotubes (MWNT) type nanofluids and $67 \%$ improvement was achieved using nano porous graphene (NPN) nanofluids. In the same study, water consumption was found to have reduced by $10 \%$ and $19 \%$ for each of the nanoparticles respectively. The study was conducted with a 0.1 weigh percentage concentration of nanofluids at 45 degrees Celsius inlet temperature.

In 2016, study on the performance of carbon nanotubes (CNT) as a transfer medium in a shell and tube cooler was done by Hosseini et al., [21]. They found that the overall heat transfer rate was increased by $10.3 \%$ and the coefficient went up by $14.5 \%$ using a $0.278 \%$ concentration. Askari et al., [18] found that three important performance parameters improved when the base fluid was replaced with a MWCNT based nanofluid. Thermal Conductivity increases by $20 \%$, the cooling range increased by $40 \%$ and the water loss is reduced by $10 \%$.

While the findings are highly encouraging, there is clearly a lack of studies investigating this type of application. Other types of nanoparticles have been studied extensively. ZnO/water, Al2O2/water, $\mathrm{Al2O} / \mathrm{EG}$, and $\mathrm{CuO}$ are among the nanofluids which also show significant promise in heat exchange application [22].

In this paper, a novel method for improvement of heat rejection performance of cooling towers by introducing nanoparticles into the base working fluid was proposed. 


\section{Methodology}

In this paper, a numerical investigation with available commercial software was chosen to simulate a various scenario with permutations of parameters. The selected cooling tower is a forced draft, cross-flow wet cooling tower with in-fill. The main interaction or functionality of the cooling tower takes place in the fill area. The fill creates a large surface area for the hot water and cooling air to interact. The main heat transfer process occurs in this zone. Hence it makes sense for the simulation to be confined to this zone for our purposes. The dimensions of a real-world cooling tower infill were determined based on the manufacturer's design data.

In this study, Multi Walled Carbon Nanotubes (MWCNT) nanoparticles was used to enhance the thermal properties of working fluid. The thermal properties of the nanoparticles are listed in Table 1.

Table 1

Characteristics of base fluid and modifying nanoparticle

\begin{tabular}{lllll}
\hline No & Properties & Units & Water & MWCNT \\
\hline 1 & Density & $\mathrm{kg} / \mathrm{m}^{\wedge} 3$ & 994 & $\sim 2100$ \\
2 & Specific Heat Capacity & $\mathrm{kJ} /(\mathrm{kg} . \mathrm{K})$ & 4.18 & $\sim 0.71$ \\
3 & Thermal Conductivity & $\mathrm{W} /(\mathrm{m} . \mathrm{K})$ & 0.6275 & $600-3000$ \\
4 & Average Diameter & $\mathrm{nm}$ & 0.275 & $10-20$ \\
\hline
\end{tabular}

Prior to the simulation, the parameters of the model and simulation have to be determined.

Based on the design and operation data of the proposed cooling tower, the following parameters were determined for the modeling. Table 2 listed the following parameters for the modeling.

\section{Table 2}

Parameters for modeling

\begin{tabular}{ll}
\hline Parameters & Value \\
\hline Velocity of Water through the Infill & $0.3 \mathrm{~m} / \mathrm{s}$ \\
Velocity of Air through the Infill & $4.54 \mathrm{~m} / \mathrm{s}$ \\
Thickness of Water Film on the Infill Plates & $0.08 \mathrm{~mm}$ \\
Spacing Between the Plates of the Infill & $32 \mathrm{~mm}$ \\
Thickness of the Infill Plates & $0.19 \mathrm{~mm}$ \\
Depth of the Infill & $850 \mathrm{~mm}$ \\
Height of the infill & $2600 \mathrm{~mm}$ \\
\hline
\end{tabular}

The parameters which were determined were then used to build the geometrical model for the simulation. The thermal properties of working fluid in the cooling tower is modified by introducing the MWCNT nanoparticles using the following equations [23-24].

Density:

$\rho_{n f}=\varphi \rho_{p}+(1-\varphi) \rho_{f}$

Thermal Conductivity:

$$
K_{n f}=\frac{2 K_{b f}+K_{p}+2 \emptyset\left(K_{p}-K_{b f}\right)}{2 K_{b f}+K_{p}-\emptyset\left(K_{p}-K_{b f}\right)} K_{b f}
$$

Specific heat capacity: 


$$
\left(\rho C_{p}\right)_{n f}=(1-\varphi)\left(\rho C_{p}\right)_{b f}+\varphi\left(\rho C_{p}\right)_{s}
$$

Dynamic Viscosity [25]:

$\mu_{n f}=\frac{\mu_{b f}}{(1-\emptyset)^{2.5}}$

In this study, one of the main parameters to be evaluated is the heat rejection by the cooling tower. The amount of heat rejection achieved by the cooling tower using the modified working fluid will determine the increase in performance of the cooling tower. Heat transfer from the heat load to the atmosphere is achieved using fluid flows. The heat content in the working fluid circulating in the cooling tower is absorbed by the cooling air interacting with it in the infill area. Based on this there will be a drop in the temperature of the working fluid between the inlet and the outlet. In industry, the following formula is used to determine the heat rejection based on the operation of the cooling tower:

$\dot{Q}=\dot{m} C_{p} \Delta T$

where

Q - heat rejection,

$\mathrm{m} \quad$ - mass flowrate of working fluid

C_p - Specific Heat Capacity of Working Fluid

$\Delta T \quad$ - $\quad$ temperature drop between inlet and outlet.

The heat rejection achieved using each working is calculated based on the cold-water outlet temperature result from the simulation. Mass flow rate is determined from the manufacturer's design data and the same value is used in the simulation model.

Based on the above equation, the thermal characteristics of the nanofluid with various fractions of nanoparticles were determined as shown in Table 3. These values were then incorporated into the materials properties in the simulation software. The new values represent physical and thermal characteristics expected of the modified working fluids i.e. nanofluids for different fractions of nanoparticles. The modified values are determined for the four fractions of nanoparticles and listed below.

Table 3

Thermal properties of nanofluids by fraction

\begin{tabular}{lllllll}
\hline Parameter & MWCNT & Water & \multicolumn{5}{l}{ Fraction of nanoparticles in fluid (\% wt.) } \\
& & & 0.01 & 0.03 & 0.1 & 0.3 \\
\hline Density, $\rho\left(\mathrm{lbm} / \mathrm{ft}^{3}\right)$ & 2100 & 998.21 & 1009.23 & 1031.26 & 1108.39 & 1328.75 \\
Specific heat, $C_{p}\left(\mathrm{~kJ} / \mathrm{m}^{3}\right)$ & 0.71 & 4.1820 & 4.1473 & 4.0778 & 3.8348 & 4.1404 \\
Thermal conductivity, $\mathrm{K}(\mathrm{W} / \mathrm{m}-\mathrm{K})$ & 3000 & 0.5984 & 0.6781 & 0.7047 & 0.7977 & 1.0635 \\
Dynamic viscosity, $\mu$ & $\mathrm{N} / \mathrm{A}$ & 0.0010016 & 0.0010271 & 0.0010808 & 0.0013034 & 0.0024 \\
\hline
\end{tabular}

As discussed previously, the interaction zone between the water and air is the most important area. A deconstructed arrangement of the cooling tower can be seen in Figure 3. From the figure, it can be seen that the entry of water into the infill area is from the top of the tower, and the air is flowing horizontal and perpendicular to both the water and the infill. The purpose of the cooling tower is achieved in this area. The infill in this model of cooling tower consists of evenly spaced vertical plates. The selected cooling tower consists of 143 fill zones or gaps between the fill pates. 


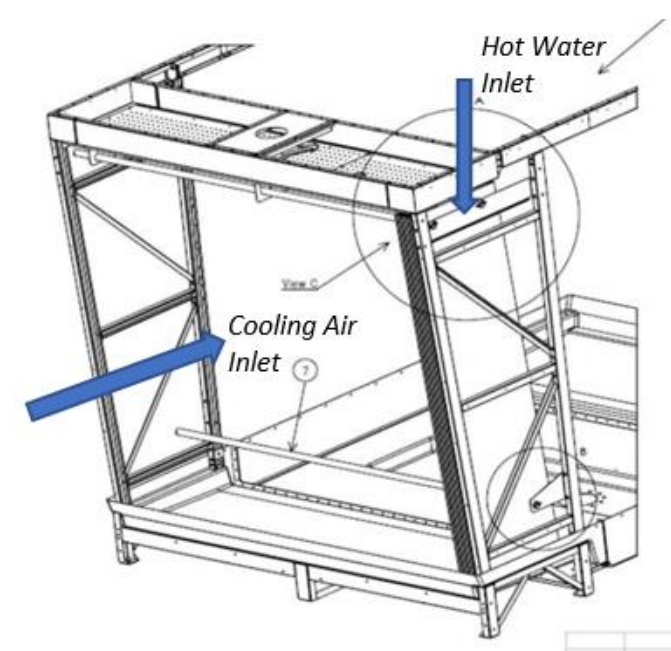

Fig. 3. Orientation of infill in cooling tower

Figure 4 shows the three-dimensional model on the simulated area. The height of the infill is $2600 \mathrm{~mm}$ while the depth is $850 \mathrm{~mm}$. Using the full dimensions of the infill area allows for accurate representation of the interaction and duration of the interaction. As the water and air flowrates are evenly distributed among the 143 fill zones, the results from the modelled fill zones can be replicated in any of the fills.

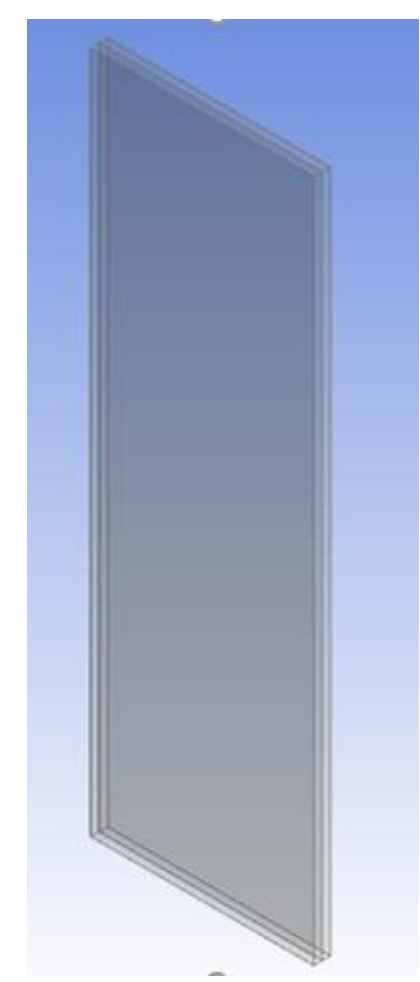

Fig. 4. Three-Dimensional model of the simulated infill

The model is generated as a Frozen body. Each zone in the model is individually defined as water or air based on the expected distribution and extruded as Sliced material. The physical boundaries and the interaction regions between the water and air layers are also defined at this stage. 
The initial conditions were also determined Based on the model and manufacturer's data. These boundary conditions are based on real operating conditions of the cooling conditions and recommended atmospheric conditions by the Malaysian Metrology Department. These boundary conditions are also used in the grid independence test before proceeding to the simulation proper. Table 4 shows the values of the initial boundary conditions.

Table 4

Initial boundary conditions

\begin{tabular}{lll}
\hline Boundary & Velocity $(\mathrm{m} / \mathrm{s})$ & Temperature $(\mathrm{K})$ \\
\hline Hot water inlet & 0.30 & 309.30 \\
Cold Air inlet & 4.54 & 306.45 \\
\hline
\end{tabular}

For the simulation to produce accurate and precise results, the mesh generated for the model must be suitable and of good quality. To determine the suitability of the mesh, a grid independence test for the various grid sizes were conducted. Ten different grid sizes were generated and tested. For the grid independence test, average the outlet water temperature for each grid size was measured from the simulation. The outlet water temperature is an important parameter in the study as this affects the heat rejection. Figure 5 shows the result of the grid independence test. It can be seen from the figure that the grid sizes below 800,000 elements show fluctuations in the trend of the water outlet temperature. However, the range of grid sizes are not suitable to solve this problem.

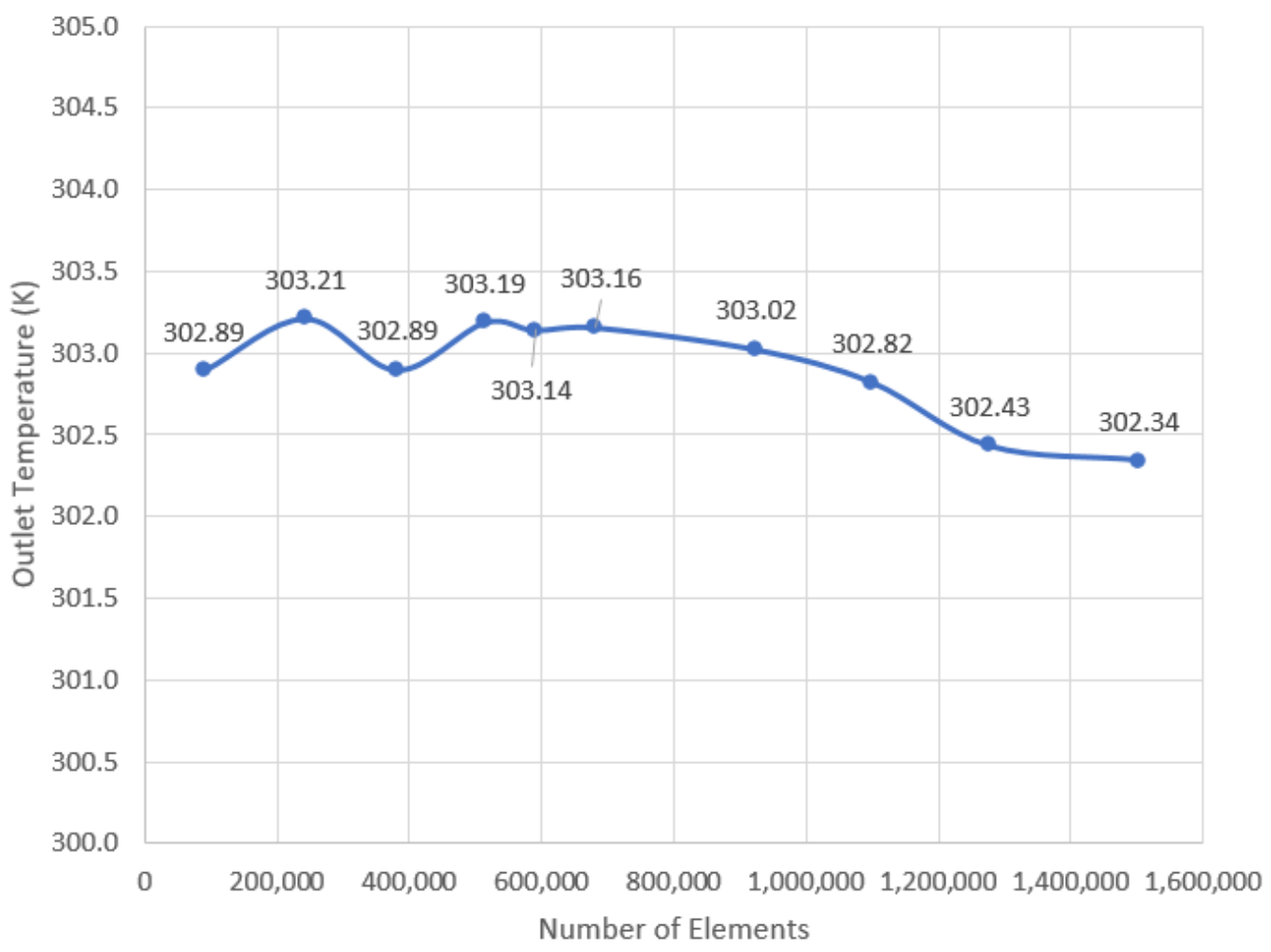

Fig. 5. Water outlet temperature for various grid sizes

The trend stabilizes beyond 800,000 elements with a consistent downturn in water outlet temperatures gradually approaching convergence around 302 Kelvin. Therefore, a grid size of $1,004,736$ elements for the simulation was chosen in this study. In addition, the Realizable k-epsilon model is found suitable to solve this problem. 


\section{Results and Discussion}

The heat transfer performance in cooling tower using modified working fluid have been conducted. As discussed briefly in the previous chapter, the simulated Heat Rejection by the cooling tower using the base fluid water as the heat transfer medium was determined to be $39,467 \mathrm{BTUH}$. This value corresponds closely to the theoretical Heat Rejection values expected based on the manufacturer's design data which is $31,217 \mathrm{BTUH}$. The parameters used in the simulation and the results are listed in Table 5 below:

Table 5

Modified parameters

\begin{tabular}{lll}
\hline Parameter & Design date & Simulation \\
\hline Density, $\rho\left(\mathrm{Ibm} / \mathrm{ft}^{3}\right)$ & 62.03 & \\
Specific heat, $C_{p}\left(\mathrm{BTU} / \mathrm{lbm}-{ }^{\circ} \mathrm{F}\right)$ & 0.9970 & \\
Volume flow rate, $v(\mathrm{ft} 3 / \mathrm{s})$ & 0.0140 & \\
Mass flow rate, $m(\mathrm{lb} / \mathrm{s})$ & 0.8698 & \\
Water inlet temperature, $\left({ }^{\circ} \mathrm{F}\right)$ & 97 & 84.36 \\
Water outlet temperature, $\left({ }^{\circ} \mathrm{F}\right)$ & 87 & 13 \\
Temperature Drop, $\Delta T\left({ }^{\circ} \mathrm{F}\right)$ & 10 & 39,467 \\
Heat Rejection, $\mathrm{Q}(\mathrm{BTUH})$ & 31,217 & \\
\hline
\end{tabular}

The difference in the theoretical and the simulated value can be attributed to safety factors. The cooling tower may be able to achieve higher heat rejection than the manufacturer's recommended operating envelope. However, manufacturer's typically design a certain percentage of buffer into the equipment to ensure the performance required by the end user is achieved without operating the equipment at $100 \%$ capacity. This difference is accompanied by an attendant variation in the coldwater outlet temperature achieved in the simulation compared to the design parameter. The manufacturer's data sheet states an outlet temperature of 87 degrees Fahrenheit. However, the simulation suggests a 3\% lower outlet temperature, 84 degrees Fahrenheit may be achievable.

For cooling towers, the common industry practice is to design a ten degrees Fahrenheit temperature drop between the hot water inlet and cold-water outlet. This provides a standard point for capacity calculations and equipment sizing. However, manufacturers are typically cautious not to propose an operating envelope that requires maximum operation capacity. Considering the percentage of discrepancy which is indeed low, we can conclude that our model and simulation are valid for these conditions. On this basis, we were able to proceed to next stage of our study which is determining heat rejection using the modified working fluid.

Table 6 listed the heat rejection achieved in the cooling tower simulation using various nanoparticle fractions in the working fluid. The table also listed the attendant parameters which were determined in order to calculate the heat rejection values. For nanofluid of $0.01 \%$ and $0.03 \%$ wt. nanoparticle fraction, there are already improvements over the base fluid. These nanofluids raise the heat rejection to 44,687 BTUH and 45,908 BTUH respectively from the baseline of 39,467 BTUH for water. There is a seemingly linear relationship between nanoparticle fraction and heat rejection in this range.

The heat rejection reaches optimal level at $0.1 \%$ wt fraction with 56,080 BTUH. However, we see a significant drop off in the heat rejection beyond this fraction. At $0.3 \%$ wt. fraction, the heat rejection is still higher than the base fluid, but less than $0.1 \%$ wt. fraction. This may be due to the effect on the 
specific heat capacity the higher fractions have. The specific heat capacity is reduced significantly, which may reduce the heat content in the working fluid despite the same temperature.

Table 6

Heat Rejection Using Nanofluids

\begin{tabular}{lllll}
\hline \multirow{2}{*}{ Parameter } & \multicolumn{4}{l}{ Fraction of Nanoparticles (\% wt.) } \\
& 0.01 & 0.03 & 0.1 & 0.3 \\
\hline Density, $\rho\left(\mathrm{lbm} / \mathrm{ft}^{3}\right)$ & 63.00 & 64.38 & 69.19 & 82.95 \\
Specific heat, $C_{p}\left(\mathrm{BTU} / \mathrm{lbm}-{ }^{\circ} \mathrm{F}\right)$ & 0.9746 & 0.9746 & 0.9165 & 0.7506 \\
Volume flow rate, $V(\mathrm{ft} 3 / \mathrm{s})$ & 0.0140 & 0.0140 & 0.0140 & 0.0140 \\
Mass flow rate, $m(\mathrm{lb} / \mathrm{s})$ & 0.8835 & 0.9028 & 0.9703 & 1.1632 \\
Water inlet temperature, $\left({ }^{\circ} \mathrm{F}\right)$ & 97 & 97 & 97 & 97 \\
Water outlet temperature, $\left({ }^{\circ} \mathrm{F}\right)$ & 82.58 & 82.51 & 79.48 & 83.63 \\
Temperature Drop, $\Delta T\left({ }^{\circ} \mathrm{F}\right)$ & 14 & 14 & 18 & 13 \\
Heat Rejection, $\mathrm{Q}(\mathrm{BTUH})$ & 44,687 & 45,908 & 56,080 & 42,037 \\
\hline
\end{tabular}

Figure 6 shows the trend in the cold-water outlet temperature and the hot air outlet temperature. It can be seen that the cold-water outlet temperature has a linear downward relationship with the increasing nanoparticle fraction up to $0.1 \%$ wt. Beyond this, it can be seen that there is no further reductions in the cold-water outlet temperature.

The operation envelope of the cooling tower is also narrower, with the heat transferred to the atmosphere lower that the smaller fractions. The temperature rise in the hot air outlet is significantly lower, which shows less heat energy has been transferred to the cooling air.

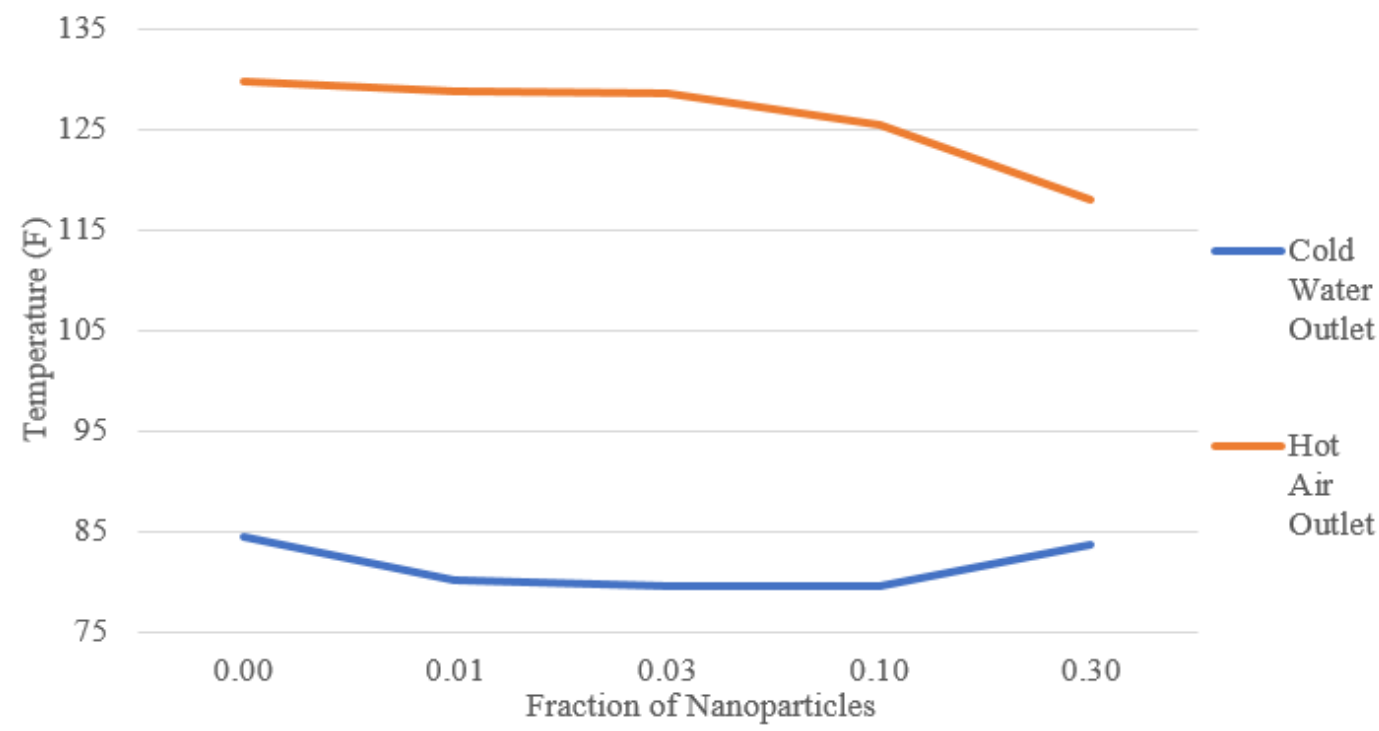

Fig. 6. Water outlet temperature for various grid sizes

The outlet temperatures for both the cold water and the hot air are close to the expected values from design data and the base fluid simulation. This further indicates that the simulation and the model are valid for this range of parameters. As discussed in the literature and in the preceding chapters, the range if the cooling tower is another common and significant parameter used in the industry to evaluate cooling tower performance. The range is the difference between the hot water inlet temperature and the cold-water outlet temperature [26]. This measure tells us what is the 
temperature reduction the cooling tower can achieve in the working fluid. This translates directly to the heat rejection to atmosphere. The range is calculated as follows:

Range $=T_{w, \text { in }}-T_{w, o u t}$

Table 7 listed the range of the cooling tower with the base fluid and the modified working fluids. The hot water inlet temperature is set to be the same for each of the case. This is the temperature based on the heat load from the heat source or building.

Table 7

Range of cooling tower with nanofluids

\begin{tabular}{llllll}
\hline \multirow{2}{*}{ Parameter } & \multicolumn{5}{c}{ Fraction of Nanoparticles (\% wt.) } \\
& Base fluid & 0.01 & 0.03 & 0.1 & 0.3 \\
\hline Water inlet temperature, $\left({ }^{\circ} \mathrm{F}\right)$ & 97 & & & & \\
Water outlet temperature, $\left({ }^{\circ} \mathrm{F}\right)$ & 84.36 & 82.58 & 82.51 & 79.48 & 83.63 \\
Range & 12.64 & 14.42 & 14.49 & 17.52 & 13.37 \\
\hline
\end{tabular}

It can be seen that the range of the cooling tower improves with the increasing fraction of nanoparticles, reaching optimal levels at $0.1 \% \mathrm{wt}$. fraction. The range for $0.3 \% \mathrm{wt}$. fraction is higher than the base fluid, but lower than the other nanofluid fractions. The various nanofluid fractions are able to achieve lower outlet water temperatures. This means the modified working fluids are more effective at transferring the heat content to the cooling air and subsequently to the atmosphere. From the data obtained through the simulation, we are able to determine several useful characteristics each nanofluid brings to the cooling tower. Table 8 listed the Reynolds Number, Nusselt Number, and the convective heat transfer coefficient for the various nanofluids.

The Reynolds number shows the flow conditions in the cooling tower of the heat transfer medium and the cooling air. With increasing nanoparticle factions, the Reynolds number is decreasing. The Reynold number in indicative of the turbulent conditions in the fluid flow. As the fluid density and dynamic viscosity increase with the nanoparticle content, the Reynolds number decreases as expected.

Table 8

Thermal characteristics

\begin{tabular}{lllll}
\hline No & Fluid & Reynold number & Nusselt number & Heat transfer coefficient \\
\hline 1 & Air & 4191 & - & - \\
2 & Water & 232 & 747 & 245.93 \\
3 & Nanofluid (0.01) & 229 & 650 & 242.29 \\
4 & Nanofluid (0.03) & 222 & 638 & 247.23 \\
5 & Nanofluid (0.10) & 198 & 555 & 243.72 \\
6 & Nanofluid (0.30) & 127 & 556 & 325.06 \\
\hline
\end{tabular}

Another interesting characteristic is the Nusselt Number, which is a ratio between the convective and conductive components of heat transfer or rejection [27]. As seen in the table above, the Nusselt number is inversely proportional to the nanoparticle fraction in the working fluid. Increasing the nanoparticle fraction produces a corresponding increase in the thermal conductivity of the fluid. It is therefore consistent to see a reduction in the Nusselt number, as the conductive component plays an increasing role in the heat rejection. 
The working fluids exhibit fluctuating values for convective heat transfer coefficient. The results indicate that there are further factors which affect the coefficient which can be investigated further. The improvement in the cooling tower performance contributes to the end user in two main ways. By improving the heat rejection capability of the cooling tower and extending its range, the energy consumption by the mechanical components is reduced. As the cooling tower is able to reject the heat more effectively from the working fluid, the flow rate to achieve the equivalent heat removal from the heat source is reduced. The circulation of the working fluid in the cooling tower circuit is achieved through pumping equipment, which consumes energy. By reducing the flow rate, this energy use is reduced. By reducing the energy used by the pumps, the cost of operation of the cooling tower system is reduced. Table 9 listed the energy consumption per month to dissipate the design heat load using the base fluid and the various nanofluid fractions. It was noting that the energy consumption for the $0.1 \% \mathrm{wt}$ fraction is the lowest as expected based on the heat rejection improvement. Using this fraction of nanofluid, the cooling tower requires $21 \%$ less energy. The lower range of nanoparticle fractions also produce significant reductions in terms of energy use. However, from the peak at $0.1 \%$ wt. fraction, we see an increase in energy consumption. Pumping of fluid requires work to be done on the fluid by the pump. For working fluids of higher nanoparticle fraction, the density of fluid increases significantly. The higher density means the pumping equipment will have to impart more work to the fluid to achieve the same flow rate.

Table 9

Energy and cost savings

\begin{tabular}{lllll}
\hline \multirow{2}{*}{ Working Fluid } & $\begin{array}{l}\text { Energy Consumption per Month } \\
(\mathrm{kWh})\end{array}$ & $\begin{array}{l}\text { Reduction in } \\
\text { Energy } \\
\text { Consumption }\end{array}$ & $\begin{array}{l}\text { Energy Cost per } \\
\text { Month } \\
(\mathrm{RM})\end{array}$ & $\begin{array}{l}\text { Cost Savings per Month } \\
(\mathrm{RM})\end{array}$ \\
\hline Water & $16,680.76$ & - & $6,088.48$ & - \\
0.01 & $14,895.09$ & $10.70 \%$ & $5,436.71$ & 651.77 \\
0.03 & $14,815.40$ & $11.18 \%$ & $5,407.62$ & 680.86 \\
0.10 & $13,035.01$ & $21.86 \%$ & $4,757.78$ & $1,330.70$ \\
0.30 & $20,846.85$ & $-24.98 \%$ & $7,609.10$ & $-1,520.62$ \\
\hline
\end{tabular}

Based on the energy consumption and reduction in energy consumption, we see that the $0.1 \%$ wt. fraction of nanofluid is able to produce a cost saving of up to RM 1,300 per month. The monetary benefit of the modified working fluid is thus clear. Furthermore, as the lower fraction nanofluids are also able to produce significant cost saving, the end user will be able to select an implementation which is suitable for their operating and expenditure conditions. The results of the simulation and the data obtained indicate that it is possible to achieve significant improvements in cooling tower performance. It can be seen that the cooling tower range can be increased with various nanoparticle fractions. The highest improvement of $38.55 \%$ increase in range was achieved at $0.1 \%$ wt. fraction. The improvements beyond that may not be feasible. Similarly, the heat rejection also saw peak improvement at $0.1 \% \mathrm{wt}$. fraction at $42 \%$. While this figure is lower than the figures seen in some literature, this is a highly significant improvement if replicated on full scale real-life applications. The impact of these improvements can be seen in Table 10, where we see a $21.86 \%$ reduction in energy consumption by the cooling tower circuit for nanoparticle fraction of $0.1 \%$ wt. Significantly, even lower fractions can achieve marked improvements in the above measures and energy consumption.

These improvements ultimately translate into monetary savings for the end user as shown in the table. A $0.1 \%$ wt. fraction nanofluid will produce up to RM1,300 in monthly saving for the user compared to using just water as the heat transfer medium. Even lower nanoparticle fractions will produce significant savings in the long run. This provides a certain flexibility to the end user in terms 
of the cost benefit balance. Different users may opt for different nanoparticle fractions depending on usage levels and cost effectiveness on implementing the modified working fluids.

Table 10

Summary of results

\begin{tabular}{lllll}
\hline \multirow{2}{*}{ Parameter } & \multicolumn{2}{l}{ Nanoparticle Fractions } & \\
& $\varnothing=0.01$ & $\varnothing=0.03$ & $\varnothing=0.1$ & $\varnothing=0.3$ \\
\hline Cooling Tower Range & $14.03 \%$ & $14.46 \%$ & $38.55 \%$ & $5.79 \%$ \\
Heat Rejection & $13.22 \%$ & $16.32 \%$ & $42.09 \%$ & $6.51 \%$ \\
Energy Consumption & $-10.70 \%$ & $-11.18 \%$ & $-21.86 \%$ & $+24.98 \%$ \\
Cost Savings per Month & RM 651.76 & RM 680.85 & RM 1,330.69 & - RM 1,520.62 \\
\hline
\end{tabular}

\section{Conclusions}

The present study was conducted to enhance the performance of cooling tower using modified working fluid. Based on computational results, the following conclusions can be made:

I. The highest improvement of thermal properties of water based MWCNT nanofluid was determined at $0.1 \%$ wt. of nanoparticles.

II. The peak performance improvement of the cooling tower using MWCNT-Water nanofluid was determined at $0.1 \%$ wt. of nanoparticles, as follow:

III. The Range of the cooling tower increased by up to $38 \%$

IV. The Heat Rejection of the cooling tower increased by up to $42 \%$

V. $21 \%$ reduction of in energy consumption cooling tower was determined at $0.1 \%$ wt. of nanoparticles.

\section{Acknowledgement}

Authors would like to acknowledge Takasago Thermal Engineering Ltd and Universiti Teknologi Malaysia (Grant no. 14B314) for supporting this research activity.

\section{References (Chicago style) Minimum 15 references}

[1] Yusof, Siti Nurul Akmal, Yutaka Asako, Mohammad Faghri, Lit Ken Tan, Nor Azwadi Bin Che Sidik, and Wan Mohd Arif Bin Aziz Japar. "Energy equation of swirling flow in a cylindrical container." International Communications in Heat and Mass Transfer 108 (2019): 104288.

https://doi.org/10.1016/j.icheatmasstransfer.2019.104288

[2] Suamir, I. Nyoman, I. Nengah Ardita, and I. Made Rasta. "Effects of Cooling Tower Performance to Water Cooled Chiller Energy Use: a Case Study toward Energy Conservation of Office Building." In 2018 International Conference on Applied Science and Technology (iCAST), pp. 712-717. IEEE, 2018.

https://doi.org/10.1109/iCAST1.2018.8751530

[3] He, Suoying, Zhiqiang Guan, Hal Gurgenci, Kamel Hooman, Yuanshen Lu, and Abdullah M. Alkhedhair. "Experimental study of the application of two trickle media for inlet air pre-cooling of natural draft dry cooling towers." Energy conversion and management 89 (2015): 644-654. https://doi.org/10.1016/i.enconman.2014.10.031

[4] Gao, Ming, Feng-zhong Sun, Kai Wang, Yue-tao Shi, and Yuan-bin Zhao. "Experimental research of heat transfer performance on natural draft counter flow wet cooling tower under cross-wind conditions." International Journal of Thermal Sciences 47, no. 7 (2008): 935-941.

https://doi.org/10.1016/j.ijthermalsci.2007.07.010

[5] Gharagheizi, Farhad, Reza Hayati, and Shohreh Fatemi. "Experimental study on the performance of mechanical cooling tower with two types of film packing." Energy conversion and management 48, no. 1 (2007): 277-280. https://doi.org/10.1016/i.enconman.2006.04.002

[6] Energy Commission. "Malaysia energy statistics handbook 2015." Kuala Lumpur: Energy Commission (2015).

[7] Markets and Markets Research Private Ltd. (2018, January 20). Cooling Tower Market. Retrieved from the Markets and Markets Research Private Ltd. Company. 
[8] IEA. World Energy Outlook 2017, IEA, Paris https://www.iea.org/reports/world-energy-outlook-2017, (2017).

[9] Li, Yingjian, Xinkui You, Qi Qiu, and Jiezhi Li. "The study on the evaporation cooling efficiency and effectiveness of cooling tower of film type." Energy conversion and management 52, no. 1 (2011): 53-59.

https://doi.org/10.1016/i.enconman.2010.06.036

[10] Goshayshi, H. R., and J. F. Missenden. "The investigation of cooling tower packing in various arrangements." Applied thermal engineering 20, no. 1 (2000): 69-80. https://doi.org/10.1016/S1359-4311(99)00011-3

[11] Hajidavalloo, Ebrahim, Reza Shakeri, and Mozaffar A. Mehrabian. "Thermal performance of cross flow cooling towers in variable wet bulb temperature." Energy Conversion and Management 51, no. 6 (2010): 1298-1303. https://doi.org/10.1016/i.enconman.2010.01.005

[12] Sadafi, M. H., I. Jahn, and K. Hooman. "Cooling performance of solid containing water for spray assisted dry cooling towers." Energy conversion and management 91 (2015): 158-167. https://doi.org/10.1016/j.enconman.2014.12.005

[13] Sidik, NA Che, and O. Adnan Alawi. "Computational investigations on heat transfer enhancement using nanorefrigerants." Journal of Advanced Research Design 1, no. 1 (2014): 35-41.

[14] Jamil, M., N. C. Sidik, and MNAW Muhammad Yazid. "Thermal performance of thermosyphon evacuated tube solar collector using TiO2/water nanofluid." Journal of Advanced Research in Fluid Mechanics and Thermal Sciences 20, no. 1 (2016): 12-29.

[15] Peyghambarzadeh, S. M., S. H. Hashemabadi, M. Seifi Jamnani, and S. M. Hoseini. "Improving the cooling performance of automobile radiator with Al2O3/water nanofluid." Applied thermal engineering 31, no. 10 (2011): 1833-1838.

https://doi.org/10.1016/i.applthermaleng.2011.02.029

[16] Imani-Mofrad, Peyman, Zeinali Heris Saeed, and Mehdi Shanbedi. "Experimental investigation of filled bed effect on the thermal performance of a wet cooling tower by using ZnO/water nanofluid." Energy conversion and management 127 (2016): 199-207.

[17] Lee, Y. K. "The use of nanofluids in domestic water heat exchanger." Journal of Advanced Research in Applied Mechanics 3, no. 1 (2014): 9-24.

[18] Askari, S., R. Lotfi, A. Seifkordi, A. M. Rashidi, and H. Koolivand. "A novel approach for energy and water conservation in wet cooling towers by using MWNTs and nanoporous graphene nanofluids." Energy conversion and management 109 (2016): 10-18. https://doi.org/10.1016/j.enconman.2015.11.053

[19] Lim, Sangwook, Hiroki Horiuchi, Alex D. Nikolov, and Darsh Wasan. "Nanofluids alter the surface wettability of solids." Langmuir 31, no. 21 (2015): 5827-5835. https://doi.org/10.1021/acs.langmuir.5b00799

[20] Bhuiyan, M. H. U., R. Saidur, M. A. Amalina, R. M. Mostafizur, and A. K. M. S. Islam. "Effect of nanoparticles concentration and their sizes on surface tension of nanofluids." Procedia Eng 105 (2015): 431-437. https://doi.org/10.1016/j.proeng.2015.05.030

[21] Hosseini, S. Masoud, Leila Vafajoo, and B. H. Salman. "Performance of CNT-water nanofluid as coolant fluid in shell and tube intercooler of a LPG absorber tower." International Journal of Heat and Mass Transfer 102 (2016): 45-53. https://doi.org/10.1016/j.ijheatmasstransfer.2016.05.071

[22] Kumar, Vikas, Arun Kumar Tiwari, and Subrata Kumar Ghosh. "Application of nanofluids in plate heat exchanger: a review." Energy conversion and management 105 (2015): 1017-1036.

https://doi.org/10.1016/i.enconman.2015.08.053

[23] Gupta, Munish, Vinay Singh, Rajesh Kumar, and Z. Said. "A review on thermophysical properties of nanofluids and heat transfer applications." Renewable and Sustainable Energy Reviews 74 (2017): 638-670.

https://doi.org/10.1016/j.rser.2017.02.073

[24] Mohammed, H. A., Husam A. Hasan, and M. A. Wahid. "Heat transfer enhancement of nanofluids in a double pipe heat exchanger with louvered strip inserts." International Communications in Heat and Mass Transfer 40 (2013): 36-46.

https://doi.org/10.1016/j.icheatmasstransfer.2012.10.023

[25] Sushobhan, B. R., and S. P. Kar. "Thermal modeling of melting of nano based phase change material for improvement of thermal energy storage." Energy Procedia 109 (2017): 385-392.

https://doi.org/10.1016/i.egypro.2017.03.035

[26] Murugaveni, S. Parimala, and P. Mohamed Shameer. "Analysis of forced draft cooling tower performance using Ansys Fluent software." International Journal of Research in Engineering and Technology 4, no. 4 (2015): $217-229$. https://doi.org/10.15623/ijret.2015.0404039 
[27] Bianco, Vincenzo, Oronzio Manca, and Sergio Nardini. "Performance analysis of turbulent convection heat transfer of Al2O3 water-nanofluid in circular tubes at constant wall temperature." Energy 77 (2014): 403-413. https://doi.org/10.1016/i.energy.2014.09.025 